\title{
The preliminary application of liver imaging reporting and data system (LI-RADS) with contrast-enhanced ultrasound (CEUS) on small hepatic nodules $(\leq 2 \mathrm{~cm})$
}

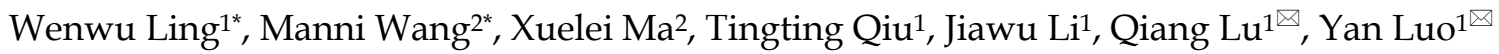 \\ 1. Department of Ultrasound, West China Hospital of Sichuan University, Chengdu 610041, China \\ 2. State Key Laboratory of Biotherapy and Cancer Center, West China Hospital of Sichuan University, Chengdu 610041, China \\ ": these authors contributed equally to this work. \\ $\bowtie$ Corresponding authors: Qiang Lu, M.D. Tel: +8628-85422304; Email: wsluqiang@126.com. Yan Luo, M.D. Tel: +8628-85423195; Email: luoyanddoc@163.com. \\ (c) Ivyspring International Publisher. This is an open access article distributed under the terms of the Creative Commons Attribution (CC BY-NC) license \\ (https://creativecommons.org/licenses/by-nc/4.0/). See http://ivyspring.com/terms for full terms and conditions.
}

Received: 2018.02.13; Accepted: 2018.07.26; Published: 2018.07.30

\begin{abstract}
To evaluate the diagnostic accuracy of liver imaging reporting and data system (LI-RADS) with contrast-enhanced ultrasound (CEUS) for patients at risk for hepatocellular carcinoma with hepatic nodules $(\leq 2 \mathrm{~cm})$. We retrospectively evaluated 56 CEUS exam records of hepatic nodules $(\leq 2 \mathrm{~cm})$ performed between January 2015 and July 2016 at West China hospital. Each nodule was classified into a LI-RADS-CEUS category by two radiologists according to imaging features. The ultimate CEUS categories were then compared with pathological reports and their correlation was then calculated. Inter-observer agreement for LI-RADS between reader $A$ and $B$ was $\mathrm{K}, 0.690$, illustrating good consistency. The diagnostic accuracy of LR- 5 on hepatocellular carcinoma (HCC) was $86.49 \%$ but $11.11 \%$ for LR-M. LI-RADS-CEUS is a potential standardized categorization system for high-risk HCC patients but might also increase the false-negative diagnosis of nodules of less than $2 \mathrm{~cm}$.
\end{abstract}

Key words: Contrast-enhanced ultrasound, Liver imaging reporting and data system, and Small hepatic nodules

\section{Introduction}

Hepatocellular carcinoma (HCC) is reported to be the sixth most common cancer worldwide [1], the incidence of which has tripled from 1975 to 2005 in the United States [2]. Patients with chronic liver disease such as cirrhosis, underlying hepatitis infection or chronic alcohol abuse are highly potential HCC groups [3]. Practice guidelines of the American Association for the Study of Liver Disease (AASLD) recommended routine imaging surveillance for patients at risk for HCC $[4,5]$ and The American College of Radiology (ACR) introduced the Liver Imaging Reporting and Data System (LI-RADS), an elaborated categorization system for patients at risk for HCC [6]. First launched in 2011, LI-RADS consists of five major categories based on the level of potential transformation to HCC.

Contrast-enhanced ultrasound (CEUS) has become one of the most commonly-used imaging techniques for modern management of liver disease.
Three separate phases are included in contrast-enhanced images: arterial, portal venous and late phase [7-9]. As is typically shown on contrast-enhanced images, HCC nodules often present hyperenhancement in the arterial phase, followed by gradual washout of the contrast agent in the portal venous and late phase. In some cases, characteristic findings on contrast-enhanced imaging allow the establishment of HCC diagnosis without liver biopsy [7]. Compared with contrast-enhanced computed tomography (CECT) and contrast-enhanced magnetic resonance imaging (CEMRI), the contrast agents used in CEUS do not diffuse into the interstitium and therefore allow real-time monitoring of vascular contrast enhancement of potentially malignant nodules which might not be fully visualized on CECT $[7,8,10,11]$. However, well-differentiated HCCs sometimes lack typical arterial hyperenhancement, appearing iso or 
even hypoenhanced in the arterial phase $[5,8]$. Another factor that might result in diagnostic inaccuracy is the influence of radiologists' subjective impression on ultrasound imaging. Standardized categorization criteria should therefore be developed to explicitly detect the likelihood of HCC.

Weighing both the advantages and disadvantages of CEUS, Arbeitsgemeinschaft der Wissenschaftlichen Medizinischen Fachgesellschaften (AWMF) and The Japanese Society of Hematology (JSH) HCC guidelines have included CEUS as a first-line diagnostic tool in the characterization of focal liver lesions [12]. As a result, a LI-RADS classification system for contrast-enhanced ultrasound (LI-RADS-CEUS) in patients at risk for HCC is devised aiming to combine the diagnostic accuracy of CEUS with the standardization of LI-RADS. Present pilot studies on LI-RADS-CEUS are very limited and in this study, we evaluated the classification accuracy of CEUS on hepatic nodules $(\leq 2 \mathrm{~cm})$ with LI-RADS.

\section{Materials and Methods}

This retrospective study was approved by institutional ethics committee and no written informed consent was required.

\section{Patient Selection}

Risk population for $\mathrm{HCC}$ was defined as patients with liver cirrhosis of any origin, hepatitis B and hepatitis C. All patients were pathologically confirmed of solid hepatic nodules $(\leq 2 \mathrm{~cm})$ and at high risk of HCC. To avoid selection bias, any clinical information of patients was not obtained before the observation process.

\section{Ultrasound examination}

The conventional ultrasound and CEUS examinations were performed with a Philips IU22 scanner (Philips Medical Solutions; Mountain View, $\mathrm{CA}$, United States) with a $1-5-\mathrm{MHz}$ convex transducer. The pulse inversion harmonic imaging was applied and the mechanical index for CEUS was 0.06. Before CEUS study, conventional ultrasound scanning was performed in advance A bolus injection of $2.4 \mathrm{~mL}$ sulfur hexafluoride-filled microbubble contrast agent (SonoVue; Bracco SpA, Milan, Italy) was administered through a 20-gauge needle placed in the antecubital vein, followed by the injection of SonoVue and a flush of $5 \mathrm{~mL} 0.9 \%$ sodium chloride solution. The timer was started as soon as the SonoVue injection completed. The target lesion and surrounding liver parenchyma were observed continuously for $6 \mathrm{~min}$.
As previously described in guidelines [13], the hepatic arterial phase was defined as the period of time starting within $20 \mathrm{~s}$ after injection and continuing for 30-45 s depending on individuals' circulatory status. The portal venous phase usually lasts for $2 \mathrm{~min}$ and the late phase continues until the complete clearance of the contrast agent from the circulation which is limited to $4-6 \mathrm{~min}$. The entire CEUS examination was stored as a dynamic digital video file on the hard disk of the ultrasound system and recorded on a digital video recorder. To record valid clips for quantification purpose, continuous scanning had to be carried out during the time of examination.

\section{Image analysis}

We retrospectively evaluated the CEUS exam records of selected patients that were performed between January 2015 and July 2016 at West China hospital. Firstly, each nodule was classified to a LI-RADS-CEUS category by two radiologists according to its imaging features on CEUS video images. Final CEUS categorization was achieved by consensus in case of different opinions. Observations were then compared to pathological reports which were designated as the reference standard.

Two Chinese Board of Radiology-certified radiologists with more than 5 years of experience in abdominal ultrasound imaging independently reviewed the contrast-enhanced ultrasonography images of the liver lesions and assigned categories based on the highest category that each lesion presented. Before the reviewing session, observers were trained following instruction on LI-RADS as was stated in previous studies [11]. Additionally, reviewing radiologists were blinded to any clinical information associated with. A preexisting category system of LI-RADS was assigned to grade each lesion and then assigned each observation into corresponding categories (LR-1, definitely benign; LR-2, probably benign; LR-3, intermediate probability for HCC; LR-4, probably HCC; LR-5, definitely HCC; LR-5V, definite tumor in vein; LR-M, probably malignant, not specific for HCC).

\section{Statistical analysis}

Inter-observer agreement was evaluated between two reviewers. Agreement was considered poor when Kappa value $<0.2$, or fair when $0.2 \leq$ Kappa value $<0.4$, or moderate when $0.4 \leq \mathrm{Kappa}$ value $<0.6$, or good when $0.6 \leq K a p p a$ value $<0.8$, or very good when $0.8 \leq K a p p a$ value $<1$. Final CEUS categorization was achieved by consensus in case of different opinions and then compared with the pathology results. MedCalc (10.4.7.0) software was used to perform the analysis. 


\section{Results}

\section{Patient and Observation Characteristics}

A total of 56 nodules in 56 patients were included in this study. Clinical information of patients including the medical history, the size, location and histopathology reports of lesions was collected during the retrospective review.

Of the 56 patients, $82.14 \%$ (46 of 56 patients) are male patients with their median age of 52.5 years. All patients were reported with chronic hepatitis or cirrhosis, including 5 with liver cirrhosis, 54 with hepatitis B virus (HBV) infection and 2 with hepatitis $\mathrm{C}$ virus (HCV) infection, but without previous history of hepatic tumors. The median diameter of nodule observations was $1.71 \mathrm{~cm}$ (minimum, $1.43 \mathrm{~cm}$; maximum, $2.00 \mathrm{~cm}$ ). Table 1 summarized relevant clinical-pathological information of 56 enrolled patients.

Table 1. Relevant clinical-pathological information of 56 enrolled patients.

\begin{tabular}{ll}
\hline Patients(n=56) & \\
\hline Age (Median) & 52.5 \\
Sex & \\
Male & $46(82.14 \%)$ \\
Female & $10(17.86 \%)$ \\
Tumor size & \\
Median & $1.71 \mathrm{~cm}$ \\
Range & $(1.43-2.00) \mathrm{cm}$ \\
Infection & \\
HBV & 54 \\
HCV & 2 \\
Pathology & \\
HCC & $44(78.57 \%)$ \\
RN & $6(10.71 \%)$ \\
ICC & $2(3.57 \%)$ \\
FNH & $2(3.57 \%)$ \\
ANG & $1(1.79 \%)$ \\
RLH & $1(1.79 \%)$ \\
\hline HBV= hepatitis B virus; HCV= hepatitis C virus. \\
HCC= hepatocellular carcinoma; RN= regenerative nodule; ICC=Intrahepatic \\
cholangiocarcinoma; FNH= focal nodular hyperplasia; ANG= angioma; RLH= \\
reactive lymphoid hyperplasia
\end{tabular}

According to histopathological reports, 44 of 56 observed patients $(78.57 \%)$ were diagnosed as HCC and $10.71 \%$ (6 of 56 patients) had regenerative nodule (RN). Intrahepatic cholangiocarcinoma (ICC), focal nodular hyperplasia (FNH), angioma (ANG) and reactive lymphoid hyperplasia (RLH) account for $3.57 \%$ ( 2 of 56 patients), $3.57 \%, 1.79 \%$ ( 1 of 56 patients) and $1.79 \%$ respectively.

\section{Distribution of categories}

Frequencies of LI-RADS categories stratified by observers are shown in Table 2.

Overall, no LR-1 and LR-2 lesions were observed by either of the observers. Thirty-seven cases of LR- 5 (66.1\% [37/56]), 8 cases of LR-4 (14.3\% [8/56]), 2 LR-3 (3.6\% [2/56]) cases and 9 LR-M $(16.0 \%[9 / 56])$ cases were identified by observer A. Meanwhile, observer B identified 37 LR-5(66.1\% [37/56]), 8 LR-4(14.3\% [8/56]), 3 LR-3 (5.3\% [3/56]) and 8 LR-M (14.3\% $[8 / 56])$.

Table 2. Frequencies of LI-RADS categories stratified by observer

\begin{tabular}{lll}
\hline LI-RADS categories & Observer A & Observer B \\
\hline LR-1 & 0 & 0 \\
LR-2 & 0 & 0 \\
LR-3 & 2 & 3 \\
LR-4 & 8 & 8 \\
LR-5 & 37 & 37 \\
LR-M & 9 & 8 \\
\hline
\end{tabular}

\section{Inter-observer Agreement}

Inter-observer agreement for CEUS LI-RADS between observer A and B was K, 0.690 (95\% CI: 0.504, 0.875), illustrating good consistency.

\section{Diagnostic Accuracy}

At last, of the 37 observations classified as LR-5, $33(89.19 \%)$ were pathologically confirmed as malignant including 1 ICC $(2.7 \%)$ and 32 HCC $(86.49 \%)$. The typical ultrasound images are shown in Figure 1. However, 4 lesions (10.81 \%) were histologically proved to be benign, 3 regenerative nodule $(8.11 \%)$ (Figure 2) and 1 focal nodular hyperplasia $(2.7 \%)$. With respect to the 8 LR-4 observations, 4 cases (50\%) turned out to be HCCs (Figure 3). Angioma, focal nodular hyperplasia (Figure 4 ) and regenerative nodule accounts for $12.5 \%$ $(1 / 8), 12.5 \%(1 / 8)$ and $25 \%(2 / 8)$ respectively. Nine observations categorized as LR-M consist of 7 $(77.78 \%)$ HCC (Figure 5), 1 (11.11\%) ICC and 1 (11.11\%) RLH, based on pathological findings. The correct diagnoses of HCC, ICC and benign lesions were used to measure diagnostic accuracy for LI-RADS-CEUS. An example of successful categorization of HCC as LR-3 was shown in Figure 6. For the diagnosis of HCC, the overall diagnostic accuracy of LR-5 was $86.49 \%(32 / 37)$ and that of LR-4 was $50 \%(4 / 8)$. However, the diagnostic accuracy of LR-M was only $11.11 \%$ in the retrospective study (Table 3).

\section{Discussion}

LI-RADS-CEUS is a newly developed guideline used to standardize the interpretation of contrast-enhanced ulstrasonography of HCCsuspicious lesions [11, 14, 15]. However, previous reports indicated that LI-RADS provided poor sensitivity and negative predictive value when maximizing its specificity $[11,16]$. In this study, we therefore evaluated the classification accuracy of hepatic nodules $(\leq 2 \mathrm{~cm})$ with Liver Imaging Reporting and Data System (LI-RADS). 

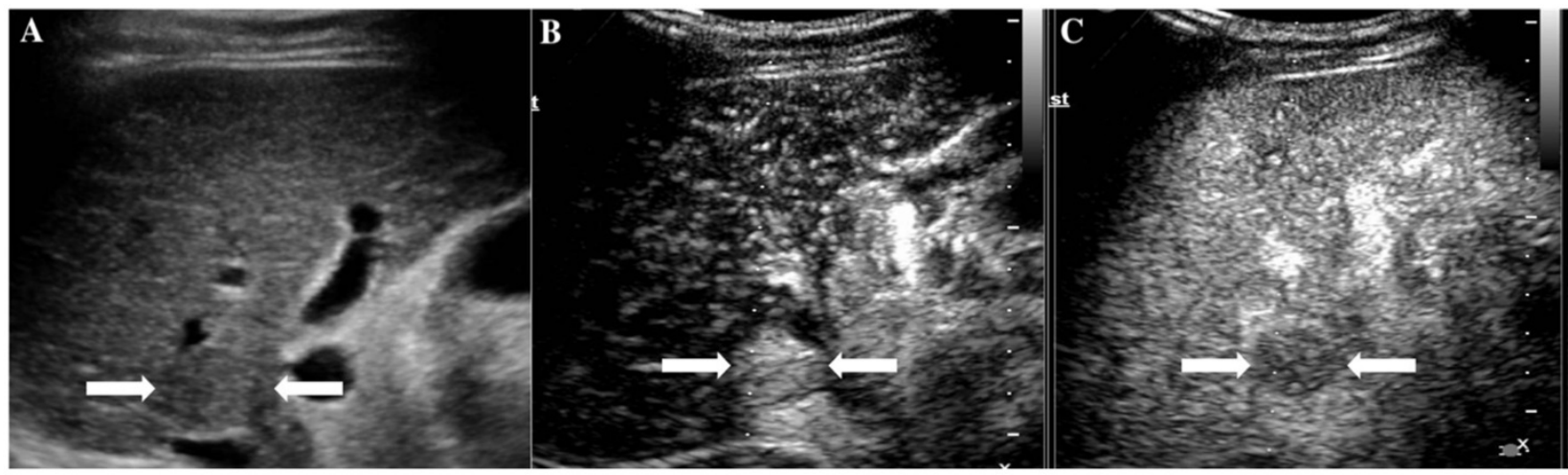

Figure 1. The assigned LI-RADS category of this patient was LR-5. Hepatocellular carcinoma (HCC) was confirmed by histopathology. A. Conventional B-mode image showed a hypoechoic nodule (arrows) of about $1.8 \mathrm{~cm}$ on the right hepatic lobe of a 52-year-old male patient. B. CEUS showed hyperenhancement (arrows) in arterial phase. C. CEUS showed hypoenhancement (arrows) in the late phase.
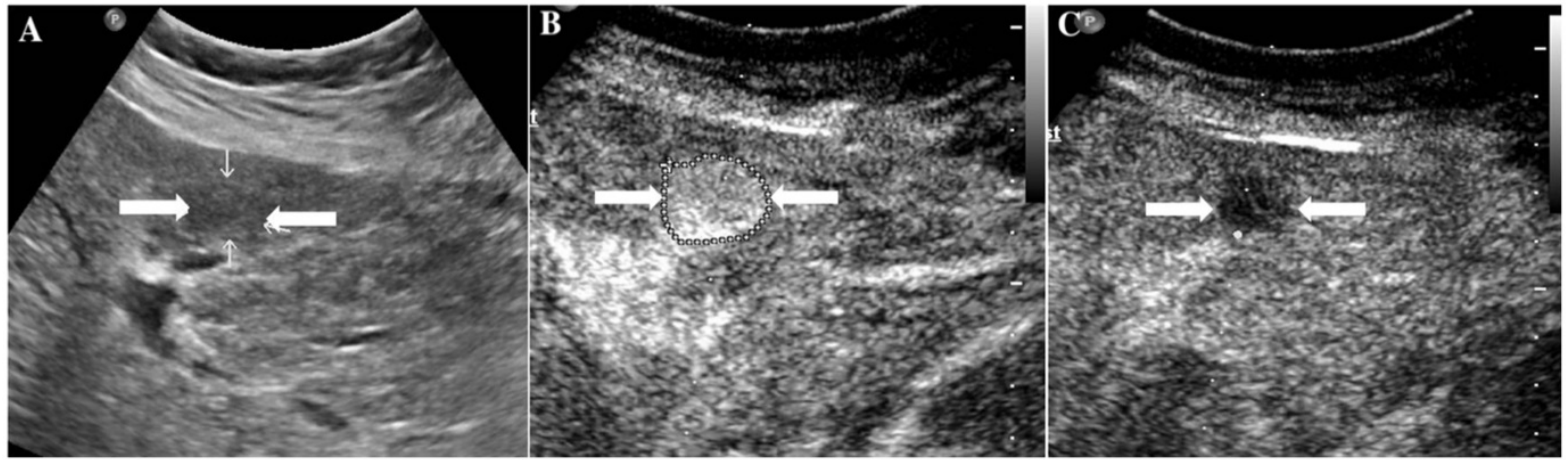

Figure 2. It illustrates an example of misinterpretation with LI-RADS-CEUS (LR-5). The ultimate pathological diagnosis was regenerative nodule (RN). A. Conventional B-mode ultrasound showed a $1.7 \mathrm{~cm}$ slightly hypoechoic nodule (arrows) on the left hepatic lobe of a 67 -year-old woman. B. CEUS showed hyperenhancement (arrows) during the arterial phase. C. CEUS showed hypoenhancement (arrows) during the late phase.
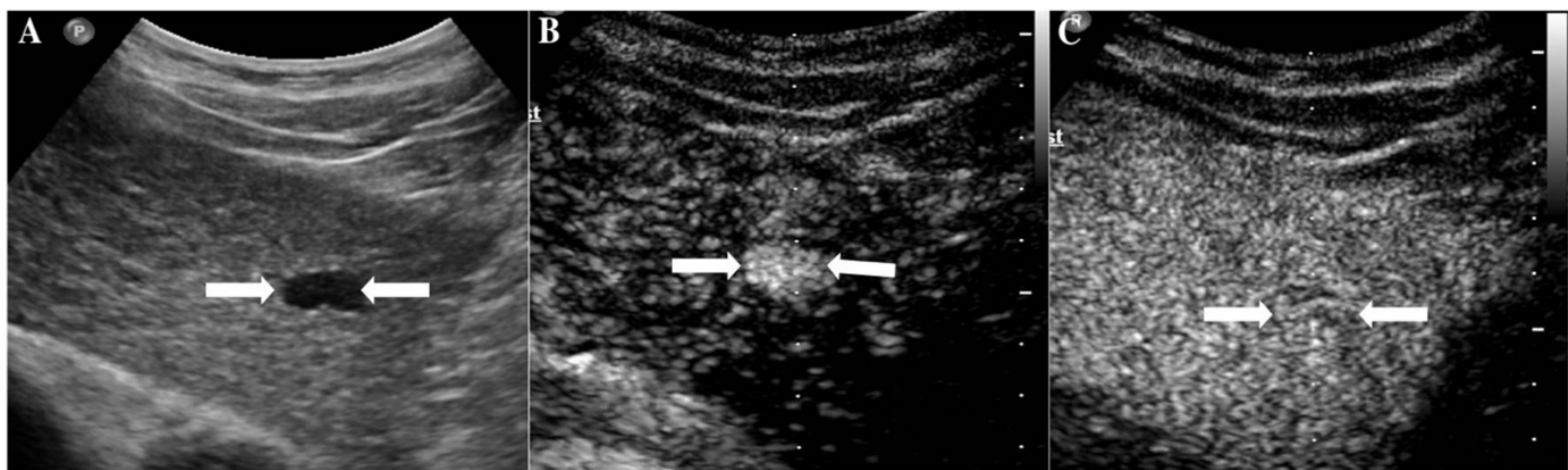

Figure 3. The assigned LI-RADS category of this patient was LR-4. Hepatocellular carcinoma (HCC) was confirmed by histopathology. A. Conventional ultrasound displayed a hypoechoic nodule (arrows) of about $1.7 \mathrm{~cm}$ on the left hepatic lobe of a 57 -year-old male patient. B. CEUS showed hyperenhancement (arrows) in arterial phase. C. CEUS showed isoenhancement (arrows) in the late phase.
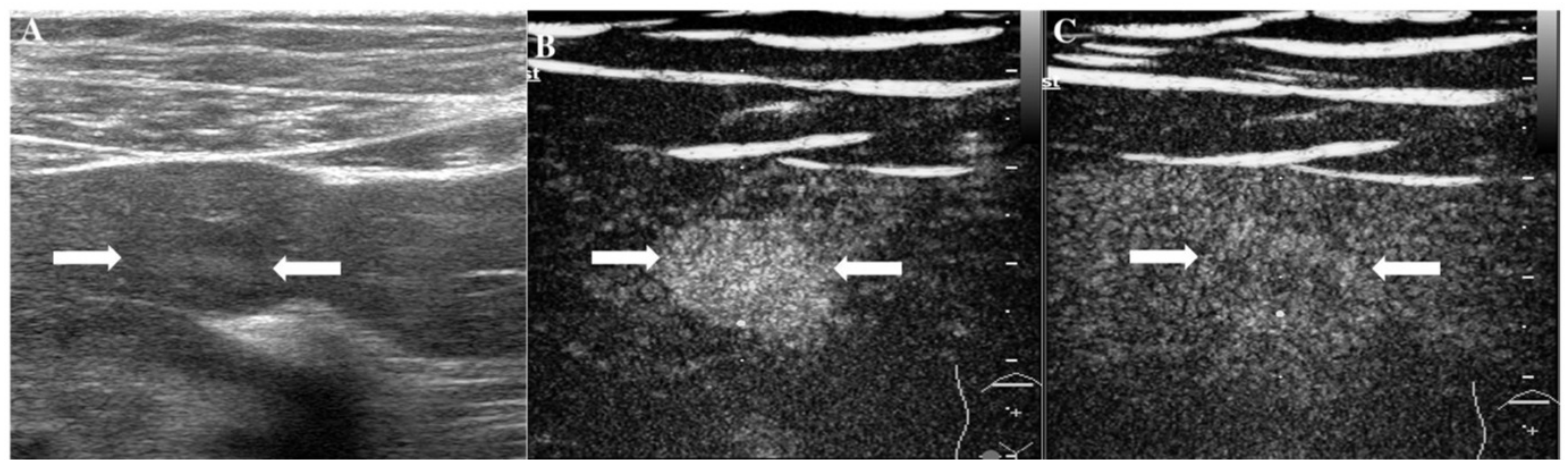

Figure 4. It illustrates an example of misinterpretation with LI-RADS-CEUS (LR-4). The ultimate pathological diagnosis was focal nodular hyperplasia (FNH). A. Conventional B-mode image showed a $1.4 \mathrm{~cm}$ isoechoic nodule (arrows) on the left hepatic lobe of a 48-year-old male. B. CEUS displayed hyperenhancement (arrows) during the arterial phase. C. CEUS showed isoenhancement (arrows) during the late phase. 

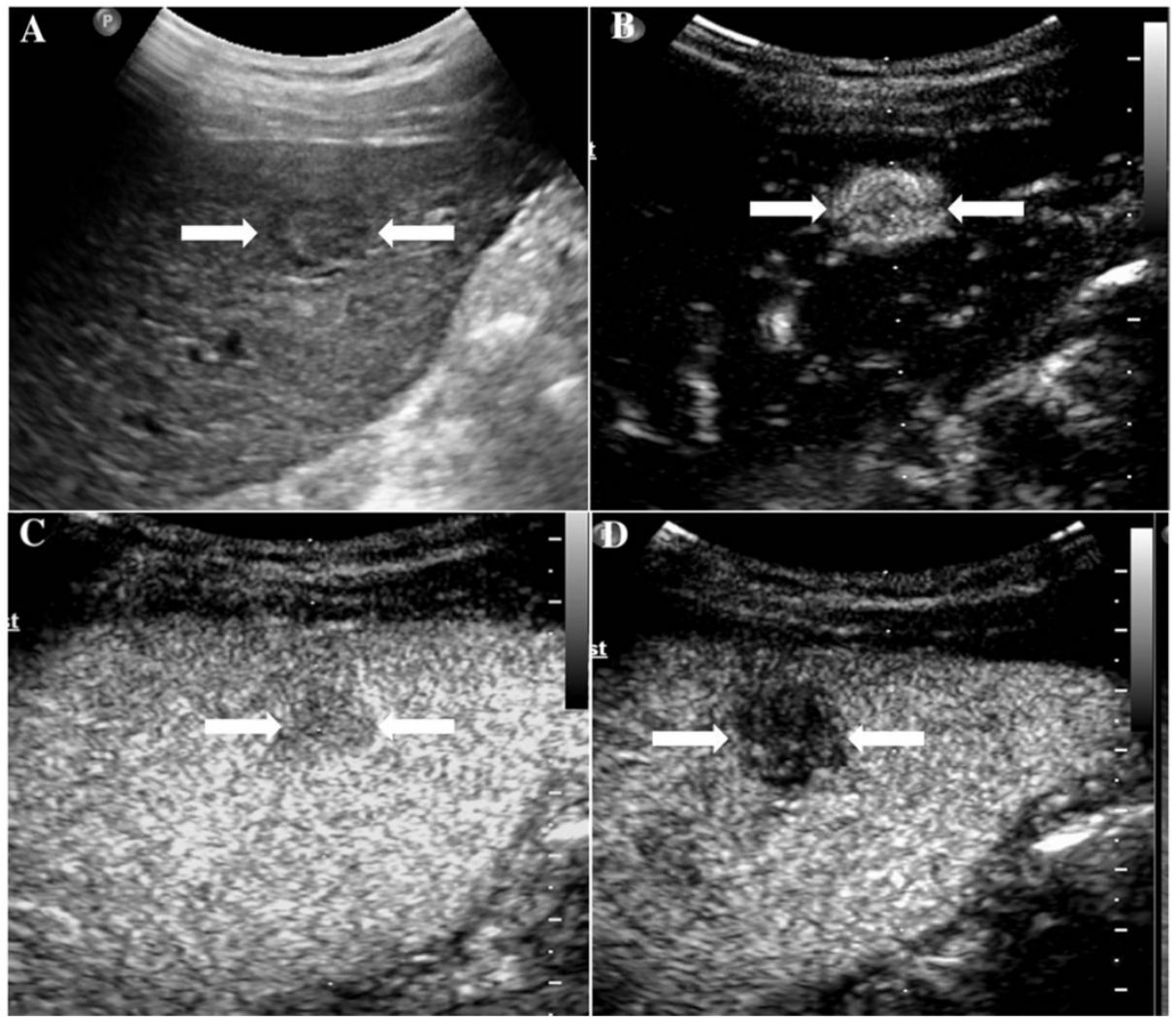

Figure 5. It illustrates an example of misinterpretation with LI-RADS-CEUS (LR-M). The ultimate pathological diagnosis was HCC. A. Conventional B-mode image showed a $2.0 \mathrm{~cm}$ hypoechoic nodule (arrows) on the right hepatic lobe of a 55-year-old male. B. CEUS showed hyperenhancement (arrows) during the arterial phase. C. The washout started after 50 seconds. D. CEUS showed obvious hypoenhancement (arrows) during the late phase.

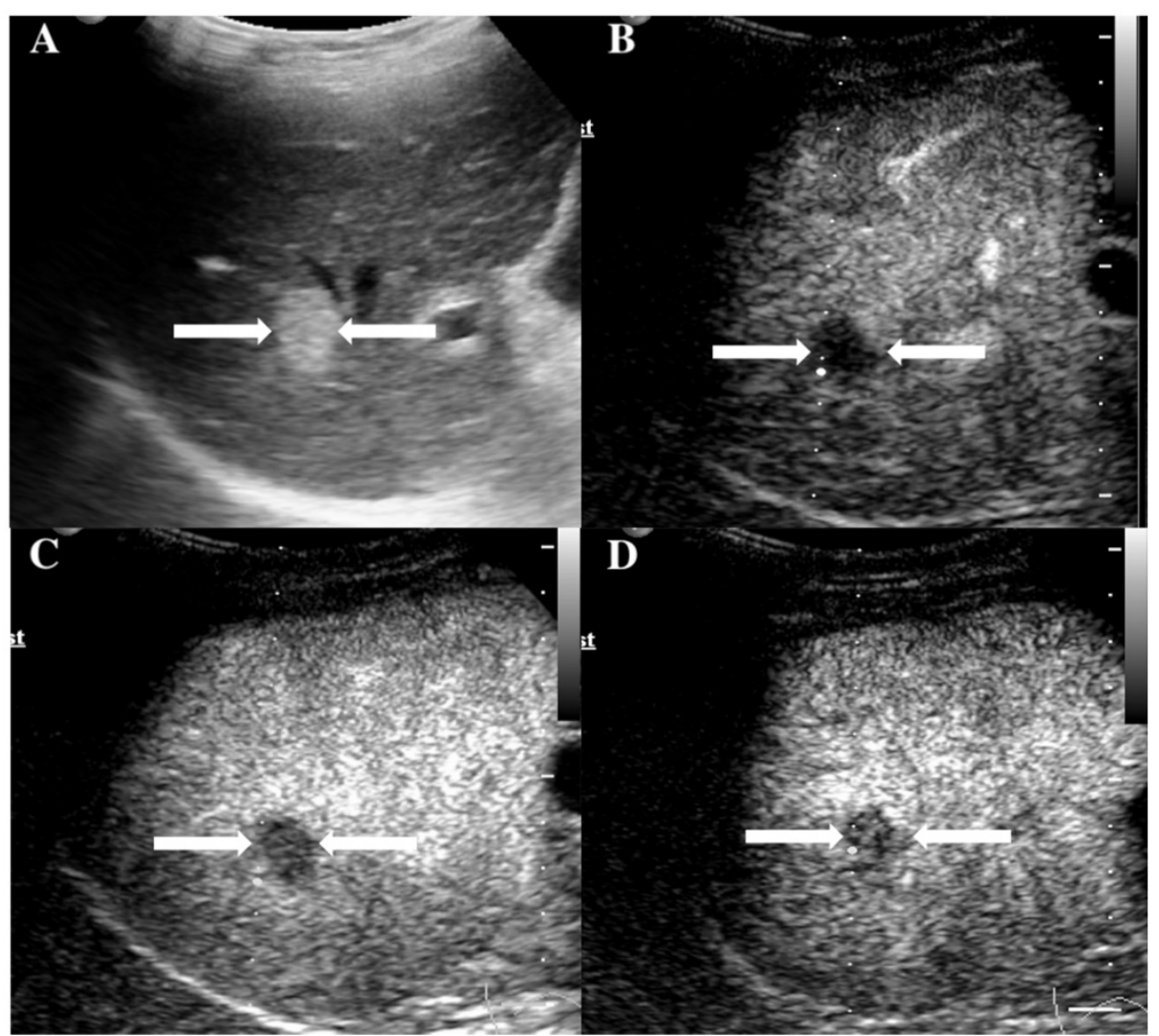

Figure 6. The assigned LI-RADS category of this patient was LR-3. Hepatocellular carcinoma (HCC) was confirmed by histopathology. A. Conventional B-mode ultrasound showed a hyperechoic nodule (arrows) of about $1.6 \mathrm{~cm}$ on the right hepatic lobe of a 36-year-old male patient. CEUS displayed hypoenhancement (arrows) in the arterial phase (Fig. 6B), portal phase (Fig. 6C) and late phase (Fig. 6D). 
Table 3. Overall diagnostic accuracy for $L R-4,5, M$

\begin{tabular}{ll}
\hline LI-RADS categories & Overall diagnostic accuracy \\
\hline LR-4 & $50 \%$ \\
LR-5 & $86.49 \%$ \\
LR-M & $11.11 \%$ \\
\hline
\end{tabular}

Inter-observer agreement among the two experienced radiologists was $\mathrm{K}, 0.690$ suggesting that the classification of small hepatic nodules with LI-RADS-CEUS is reproducible with good consistency in patients with chronic liver disease. The majority of nodules classified as LR- 5 turned out to be HCC at pathological diagnosis, but some ICCs, regenerative nodules and focal nodular hyperplasia could also illustrate hyper enhancement at the arterial phase on CEUS. Similar conclusion could be drawn about LR-4 nodules that most LR-4 nodules were pathologically confirmed of HCC. Whereas $50 \%$ (4/8) of LR-4 lesions turned out to be benign in the end (1 angioma, 1 focal nodular hyperplasia and 2 hyperplastic nodules). Last but not least, 7 out of 9 LR-M lesions turned out to be HCC with the diagnostic accuracy of only $11.11 \%$. LR-M refers to potentially malignant nodules other than HCCs, with wash-out characteristics of early wash-out onset $(\leq 60 \mathrm{~s})$. This wash-out characteristic would increase the false-negative diagnosis of nodules of less than $2 \mathrm{~cm}$ in diameter. According to our experience, the onset of wash-out of metastatic lesion is in early portal phase, even in the late arterial phase in some cases. Therefore, the threshold of wash out onset for the categorization of LR-M lesions needs further verification. Small hepatic lesions present diagnostic challenge especially in cirrhotic liver, with limited sensitivity not only on ultrasonography but also a problem computed tomography (CT) and magnetic resonance imaging (MRI) [17].

CEUS was first brought out in 2005 AASLD guidelines as one major characterization techniques of potential HCC lesions, together with CECT and CEMRI. However, it was later excluded from AASLD and EASL guidelines in 2011 and 2012 due to some arguments about its failure to differentiate HCC from ICC [18-20]. The Erlangen group from Germany proposed a separate category for classification of ICCs in 2016 [21, 22]. Previous literatures have studied certain enhancement patterns of ICC and HCC based on radiological observations and a new category was accordingly designed to distinguish ICCs from HCCs $[13,23]$. On the other hand, CEUS was reported to be helpful in identifying BI-RADS category 3 or 4 small breast lesions, suggesting the potential utility of CEUS in categorizing small hepatic nodules [24].

Certain limitations about LI-RADS-CEUS do exist and yield more solutions. Leoni et al. [7] concluded that CEUS sensitivity improves with omission of wash-out time as a diagnostic criterion, which corresponded to our discovery that small HCC lesions $(\leq 2 \mathrm{~cm})$ with early onset of wash-out $(\leq 60 \mathrm{~s})$ were often mistakenly categorized into LR-M. Nonetheless, LI-RADS-CEUS designated ICC patients should be provided with adequate histological validation of dysplastic and regenerative nodules to determine whether CEUS enables reliable differentiation of ICCs from HCCs. Another difficulty is the differential diagnosis between benign nodules and early HCCs, which is in accordance with a recent study evaluating LI-RADS in 159 cirrhotic patients [20]. Considering the transition between multiple stages of carcinogenesis in HCCs, the identification of intermediate categories such as LR-2, 3 and 4 remains challenging. Furthermore, although our study reflected a good inter-observer agreement, some studies have revealed difficulties in the observation of some specific imaging features consistently among the different readers [5]. Since our study only enrolled patients with surgery or biopsy pathological reports and LR-1, 2 are usually small lesions, they normally do not undergo puncture or surgery. So it is difficult to evaluate LR-1 and LR-2 patients.

In conclusion, LI-RADS-CEUS presented good inter-observer agreement but might at the same time increase the false-negative diagnosis of nodules of less than $2 \mathrm{~cm}$ in diameter in patients with chronic liver disease. LI-RADS-CEUS bears the potential to be promoted as a standardized category system for liver observations in high-risk HCC patients after refinement of its preexisting problems addressed in this study.

\section{Abbreviations}

LI-RADS: liver imaging reporting and data system; CEUS: contrast-enhanced ultrasound; LI-RADS-CEUS: liver imaging reporting and data system- contrast-enhanced ultrasound; HCC: hepatocellular carcinoma; AASLD: American Association for the Study of Liver Disease; ACR: American College of Radiology; CECT: contrast-enhanced computed tomography; CEMRI: contrast-enhanced magnetic resonance imaging; AWMF: Arbeitsgemeinschaft der Wissenschaftlichen Medizinischen Fachgesellschaften; JSH: Japanese Society of Hematology; HBV: hepatitis B virus; HCV: hepatitis $\mathrm{C}$ virus; $\mathrm{RN}$ : regenerative nodule; ICC: intrahepatic cholangiocarcinoma; FNH: focal nodular hyperplasia; ANG: angioma; RLH: reactive lymphoid hyperplasia; CT: computed tomography; MRI: magnetic resonance imaging. 


\section{Acknowledgements}

This work was supported by the National Natural Science Foundation of China (No.81501488), Sichuan Science and Technology Program (No.2017JY0267) and Chengdu Science and Technology Bureau (No.2014-HM01-00021-SF).

\section{Competing Interests}

The authors have declared that no competing interest exists.

\section{References}

1. Bray F, Ren JS, Masuyer E, et al. Global estimates of cancer prevalence for 27 sites in the adult population in 2008. Int J Cancer. 2013;132:1133-45.

2. Altekruse SF, McGlynn KA, Reichman ME. Hepatocellular carcinoma incidence, mortality, and survival trends in the United States from 1975 to 2005. J Clin Oncol. 2009;27:1485-91.

3. Llovet JM, Burroughs A, Bruix J. Hepatocellular carcinoma. Lancet. 2003;362:1907-17.

4. Petruzzi N, Mitchell D, Guglielmo F, et al. Hepatocellular carcinoma likelihood on MRI exams: evaluation of a standardized categorization system. Acad Radiol. 2013;20:694-8.

5. Zhang YD, Zhu FP, Xu X, et al. Classifying CT/MR findings in patients with suspicion of hepatocellular carcinoma: Comparison of liver imaging reporting and data system and criteria-free Likert scale reporting models. J Magn Reson Imaging. 2016;43:373-83.

6. Elsayes KM, Hooker JC, Agrons MM, et al. 2017 Version of LI-RADS for CT and MR Imaging: An Update. Radiographics. 2017;37:1994-2017.

7. Leoni S, Piscaglia F, Granito A, et al. Characterization of primary and recurrent nodules in liver cirrhosis using contrast-enhanced ultrasound: which vascular criteria should be adopted? Ultraschall Med. 2013;34:280-7.

8. Claudon M, Dietrich CF, Choi BI, et al. Guidelines and good clinical practice recommendations for contrast enhanced ultrasound (CEUS) in the liver--update 2012: a WFUMB-EFSUMB initiative in cooperation with representatives of AFSUMB, AIUM, ASUM, FLAUS and ICUS. Ultraschall Med. 2013;34:11-29.

9. Friedrich-Rust M, Klopffleisch T, Nierhoff J, et al. Contrast-Enhanced Ultrasound for the differentiation of benign and malignant focal liver lesions: a meta-analysis. Liver Int. 2013;33:739-55.

10. Westwood M, Joore M, Grutters J, et al. Contrast-enhanced ultrasound using SonoVue ${ }^{\circledR} \quad$ (sulphur hexafluoride microbubbles) compared with contrast-enhanced computed tomography and contrast-enhanced magnetic resonance imaging for the characterisation of focal liver lesions and detection of liver metastases: a systematic review and cost-effectiveness analysis. Health Technol Assess. 2013;17:1-243.

11. Mitchell DG, Bruix J, Sherman M, et al. LI-RADS (Liver Imaging Reporting and Data System): summary, discussion, and consensus of the LI-RADS Management Working Group and future directions. Hepatology. 2015;61:1056-65.

12. Kokudo N, Hasegawa K, Akahane M, et al. Evidence-based Clinical Practice Guidelines for Hepatocellular Carcinoma: The Japan Society of Hepatology 2013 update (3rd JSH-HCC Guidelines). Hepatol Res. 2015;45:123-7.

13. D'Onofrio M, Crosara S, De Robertis R, et al. Contrast-Enhanced Ultrasound of Focal Liver Lesions. AJR Am J Roentgenol. 2015;205:W56-66.

14. Purysko AS, Remer EM, Coppa CP, et al. LI-RADS: a case-based review of the new categorization of liver findings in patients with end-stage liver disease. Radiographics. 2012;32:1977-95.

15. Santillan CS, Tang A, Cruite I, et al. Understanding LI-RADS: a primer for practical use. Magn Reson Imaging Clin N Am. 2014;22:337-52.

16. Tang A, Valasek MA, Sirlin CB. Update on the Liver Imaging Reporting and Data System: What the Pathologist Needs to Know. Adv Anat Pathol. 2015;22:314-22.

17. Strobel $\mathrm{D}$, Bernatik $\mathrm{T}$, Blank $\mathrm{W}$, et al Diagnostic accuracy of CEUS in the differential diagnosis of small $(\leq 20 \mathrm{~mm})$ and subcentimetric $(\leq 10 \mathrm{~mm})$ focal liver lesions in comparison with histology. Results of the DEGUM multicenter trial. Ultraschall Med. 2011;32:593-7.

18. Wildner D, Bernatik T, Greis C, et al. CEUS in hepatocellular carcinoma and intrahepatic cholangiocellular carcinoma in 320 patients - early or late washout matters: a subanalysis of the DEGUM multicenter trial. Ultraschall Med. 2015;36:132-9.

19. Bota S, Piscaglia F, Marinelli S, et al. Comparison of international guidelines for noninvasive diagnosis of hepatocellular carcinoma. Liver Cancer. 2012:1:190-200.

20. Darnell A1, Forner A, Rimola J, et al. Liver Imaging Reporting and Data System with MR Imaging: Evaluation in Nodules $20 \mathrm{~mm}$ or Smaller Detected in Cirrhosis at Screening US. Radiology. 2015;275:698-707.
21. Schellhaas B, Görtz RS, Pfeifer L, et al. Diagnostic accuracy of contrast-enhanced ultrasound for the differential diagnosis of hepatocellular carcinoma: ESCULAP versus CEUS-LI-RADS. Eur J Gastroenterol Hepatol. 2017;29:1036-44

22. Schellhaas B, Wildner D, Pfeifer L, et al. LI-RADS-CEUS - Proposal for a Contrast-Enhanced Ultrasound Algorithm for the Diagnosis of Hepatocellular Carcinoma in High-Risk Populations. Ultraschall Med. 2016;37:627-34.

23. Egger C, Goertz RS, Strobel D, et al. Dynamic contrast-enhanced ultrasound (DCE-US) for easy and rapid evaluation of hepatocellular carcinoma compared to dynamic contrast-enhanced computed tomography (DCE-CT)--a pilot study. Ultraschall Med. 2012;33:587-92.

24. Jang HJ, Kim TK, Burns PN, et al. CEUS: An essential component in a multimodality approach to small nodules in patients at high-risk for hepatocellular carcinoma. Eur J Radiol. 2015; 84:1623-35. 